\title{
Recognizing Factitious Hypoglycemia in the Family Practice Setting
}

\author{
Cynthia M. Waickus, $M D, P b D$, Andrée de Bustros, $M D$, and Amer Shakil, MD
}

Background: Factitious hypoglycemia is a deliberate attempt to induce a low serum glucose level using either insulin or oral hypoglycemic agents. Sulfonylurea-induced hypoglycemia is more common than incidents of insulin abuse, and hypoglycemia caused by these oral agents is biochemicallyindistinguishable from insulinoma.

Methods: We describe a case of factitious hypoglycemia resulting from insulin abuse in an adult diabetic patient, review the essentials of glucose homeostasis, and describe diagnostic tests that allow a differential diagnosis.

Results and Conclusion: Factitious hypoglycemia is associated with a higher incidence of suicide, depression, and personality disorders. Insulin-induced hypoglycemia can be detected by an insulin to $\mathrm{C}$-peptide ratio that is greater than 1.0. In the absence of proof to the contrary, insulinoma should be considered the cause of hypoglycemia until another diagnosis is established. The generally poor prognosis for patients with factitious hypoglycemia underscores the importance of early recognition of factitious disorders. (J Am Board Fam Pract 1999;12:133-6.)

Hypoglycemia is a potentially fatal condition that is routinely treated in emergency departments and is often encountered by family physicians. Factitious hypoglycemia ${ }^{1}$ is a psychiatric disorder in which a patient deliberately manipulates serum glucose by insulin ${ }^{2,3}$ or sulfonylurea abuse ${ }^{4}$ to induce illness. Factitious disorders, in general, are referred to as Munchausen syndrome, and can be a major diagnostic challenge because the symptoms are often real and the patient is actively striving to deceive the physician. ${ }^{1}$ The laboratory profile of factitious hypoglycemia and that of hypoglycemia secondary to a covert insulinoma overlap considerably, and the expedient diagnosis of a factitious disorder might obviate the need for a lengthy and arduous search for an insulinoma. We describe a case of factitious hypoglycemia resulting from insulin overdose and review the differential diagnosis of hypoglycemia in this setting.

Submitted, revised, 29 June 1998.

From the Department of Family Medicine (CMW),RushPresbyterian-St. Luke's Medical Center, Chicago, the Department of Internal Medicine (AB), Christ Hospital and Medical Center, Oak Lawn, and the UIC-Christ Family Practice Residency Program (AS), Christ Hospital/University of Illinois at Chicago, Oak Lawn, Ill. Address reprint requests to Cynthia $M$. Waickus, $\mathrm{MD}, \mathrm{PhD}$, Department of Family Medicine, Rush-Presbyterian-St. Luke's Medical Center, Chicago, IL 60612.

\section{Methods}

A case of factitious hypoglycemia is described that resulted from insulin abuse in an adult diabetic patient admitted to the hospital through the emergency department. We present a brief literature review of glucose homeostasis and insulin metabolism along with diagnostic tests that allow a differential diagnosis between insulinoma and factitious hypoglycemia.

\section{Case Report}

A 39-year-old woman with insulin-requiring diabetes mellitus came to the emergency department at a tertiary care center with symptoms of diaphoresis, nausea, and one episode of vomiting. The patient reported persistent hypoglycemia lasting several days; on the day of admission her blood glucose, by home finger stick, was 39 $\mathrm{mg} / \mathrm{dL}$ and did not increase following ingestion of juice or candy. Findings of a physical examination in the emergency department were unremarkable except for dry mucous membranes. Her blood glucose level on admission was $30 \mathrm{mg} / \mathrm{dL}$; she was given juice and her blood glucose level increased to $40 \mathrm{mg} / \mathrm{dL}$. She was given an ampule of 50 percent dextrose, a 5 percent dextrose solution was started intravenously, and she was admitted to the hospital. She denied the use of insulin or glucose- 
Table 1. Common Symptoms and Signs of Hypoglycemia.

\begin{tabular}{ll}
\hline Neuroglycopenic & Autonomic \\
\hline Headache & Diaphoresis \\
Blurred or double vision & Tremor \\
Confusion or slow mentation & Anxiety \\
Focal central nervous system & Palpitations \\
signs (diplopia, hemiparesis) & Hunger \\
Odd behavior & Paresthesias \\
Hypothermia & \\
Convulsions & \\
Coma & \\
\hline
\end{tabular}

lowering agents for at least the 3 previous days.

One week before this episode she had been admitted to another hospital with symptoms of hypoglycemia. When health care workers at the previous hospital were contacted, they said they suspected self-administration of insulin, but no proof was found; she was given glucose and was discharged with her plasma glucose level close to normal.

During the next few days in our hospital, her blood glucose remained between 40 and 60 $\mathrm{mg} / \mathrm{dL}$, and she was maintained on $1 \mathrm{~L}$ of intravenous 5 percent dextrose solution every 6 hours. She also required multiple injections of 50 percent dextrose solution because of lethargy and was provided with an ample supply of bedside juices and candy; her blood glucose level was monitored every 1 to 2 hours. A pattern of low plasma glucose values was observed that, compared with daytime values, was more prominent at night. On the fifth day of hospitalization, a very high level of insulin was detected in the patient's blood (525 $\mu \mathrm{U} / \mathrm{mL}$; normal 5 to $25 \mu \mathrm{U} / \mathrm{mL}$ ). Further laboratory tests indicated a low C-peptide level of 0.4 $\mathrm{ng} / \mathrm{mL}$ (normal 0.8 to $4.0 \mathrm{ng} / \mathrm{mL}$ ) and no traces of sulfonylureas, anti-insulin, or insulin receptor antibodies. The insulin to $\mathrm{C}$-peptide molar ratio was markedly elevated (695).

The patient was confronted with the possibility of insulin abuse, but she persistently denied it. A search of her hospital bathroom, however, uncovered a large supply of insulin and two prefilled syringes hidden within the installed toilet tissue roll. She repeatedly denied insulin use and attempted to leave the hospital against medical advice. The patient was transferred to medical intensive care unit because of the possibility that she had in-
Table 2. Hormonal Control of Serum Glucose Levels.

\begin{tabular}{|c|c|c|c|}
\hline Hormone & Glycogenolysis & Gluconeogenesis & Lipolysis \\
\hline Insulin & $\downarrow$ & $\downarrow$ & $\downarrow$ \\
\hline Glucagon & $\uparrow \uparrow$ & $\uparrow \uparrow$ & $\uparrow \uparrow$ \\
\hline Epinephrine & $\uparrow \uparrow \uparrow$ & 0 & $\uparrow \uparrow$ \\
\hline Growth hormone & 0 & 0 & $\uparrow$ \\
\hline Glucocorticoids & 0 & $\uparrow \uparrow$ & $\uparrow$ \\
\hline
\end{tabular}

jected dangerous amounts of insulin. While there, her blood glucose returned to normal levels dur- $\overrightarrow{0}$ ing the course of 2 days and later climbed to her ${ }_{\vec{t}}^{\omega}$ normal range. Psychiatric examination concluded N the patient was depressed, but not suicidal, ando음 she was later transferred to a psychiatric unit.

\section{Discussion \\ Glucase Homeastasis}

Hypoglycemia is defined as low plasma glucose levels (less than 45 to $50 \mathrm{mg} / \mathrm{dL}$ in men, and less than 35 to $40 \mathrm{mg} / \mathrm{dL}$ in women) manifesting symptoms that improve in response to carbohy- $\overrightarrow{0}$ drate administration (the so-called Whipplece triad). Common symptoms and signs of hypoglycemia $^{5}$ are shown in Table 1. Hypoglycemia is often categorized as fasting (postabsorptive) and reactive (postprandial). It is commonly thought ${ }_{\Omega}^{\circ}$ that fasting hypoglycemia implies a serious disor- $\overrightarrow{\overrightarrow{0}}$ der, such as insulinoma, whereas reactive hypoglycemia is invariably benign. This distinction is true in general, although patients with serious hypoglycemic disorders can also have postprandial $\sum_{\Sigma}$ hypoglycemia. To understand the differential di- $-\frac{\dot{\AA}}{\partial}$ agnosis of hypoglycemia, a brief review of glucose metabolism is helpful.

Normoglycemia is achieved by the interaction 9 of several hormones, including insulin, glucagon, No epinephrine, cortisol, and growth hormone. In $>$ the fasting state, insulin secretion is suppressed and counterregulatory mechanisms are activated. Glucagon and epinephrine participate in acute, short-term regulation of serum glucose, whereas cortisol and growth hormone are involved in long-term regulation. The actions of these agents $?$ on glycogenolysis, gluconeogenesis, and lipolysis 5 are shown in Table 2.

After meals or after an intravenous load of glu- $\frac{\mathbb{\infty}}{2}$ cose, islet cells release insulin. Insulin stimulates glucose entry into cells where it is utilized as fuel; 
Table 3. Differential Diagnosis of Hypoglycemia.

\begin{tabular}{lccc}
\hline Diagnosis & Glucose Level & Insulin Level & C-Peptide Level \\
\hline Normal person & Normal & Normal & Normal \\
& $(>40 \mathrm{mg} / \mathrm{dL})$ & $(<6 \mu \mathrm{mL})$ & $(<0.2 \mathrm{mmol} / \mathrm{L})$ \\
Endogenous insulin (insulinoma) & Low & Elevated & Elevated \\
Exogenous insulin administration & Low & Elevated & Low-undetectable \\
Sulfonylurea overdose & Low & Elevated & Elevated \\
Coexisting adrenal or pituitary disease & Low-normal & Low-normal & Low-normal \\
Non-insulin-mediated hypoglycemia & Low-normal & Low-normal & Low-normal \\
(insulinlike factors) & & &
\end{tabular}

excess glucose is used to make glycogen. The complex regulatory mechanisms can fail if (1) insulin or insulin-like factors continue to be produced in the face of hypoglycemia (insulinoma, or sulfonylurea abuse, tumors secreting insulinlike substances), (2) counterregulatory hormone activity fails (adrenal or pituitary insufficiency), or (3) substrates are unavailable (liver dysfunction, alcohol abuse). When glycogen stores are depleted or are unavailable because of one of the factors stated previously ( 2 or 3 ), in healthy persons the protein stores and triglyceride oxidation provide the liver with substrate materials (alanine and fatty acids) for gluconeogenesis. After prolonged fasting (typically longer than 72 hours), ketone bodies are produced to provide the central nervous system with a continuous glucose supply. ${ }^{5} \mathrm{By}$ contrast, in states of insulin excess (1, above), gluconeogenesis, lipolysis, fatty acid oxidation, and ketone production are inhibited.

\section{Insulin Metabolism}

Proinsulin, secreted from beta cells, is converted to insulin and $C$ peptide and released into the bloodstream in equimolar amounts. ${ }^{6}$ Endogenous insulin is metabolized by the liver, whereas $C$ peptide is slowly cleared by the kidney and is not metabolized much in the liver. As a result, hypoglycemia associated with increased endogenous insulin secretion (more than $6 \mu \mathrm{U} / \mathrm{mL}$ ) is accompanied by inappropriately elevated C-peptide levels (in excess of $0.2 \mathrm{mmol} / \mathrm{L}$ ). Exogenous insulin administration, however, will always result in much higher levels of insulin and very low to undetectable levels of $\mathrm{C}$ peptide. Fasting hypoglycemia in the face of other coexisting endocrine (ie, pituitary or adrenal) disease typically requires little diagnostic investigation of the hypoglycemia. Because these disorders are generally multisymp- tomatic, recognizing the underlying disease and its associated risk and treatment for hypoglycemia is sufficient. $3,6-8$

Hypoglycemia is often caused by accidental overdoses in patients receiving sulfonylureas and often arises from inadvertent administration of soundalike medications (ie, acetohexamide for acetazolamide) or errors in self-dosing. ${ }^{4} \mathrm{Hypo}-$ glycemia resulting from sulfonylurea overdose is much more common than insulin-induced factitious hypoglycemia, ${ }^{4}$ most likely because of the widespread availability of the oral prescriptions. Hypoglycemia caused by oral agents is biochemically indistinguishable from insulinoma (C-peptide and insulin levels are both increased; the insulin to C-peptide molar ratio is less than 1.0) but can be detected by specific chemical assay of sulfonylureas in the serum or urine. Insulinoma should be considered the cause of hypoglycemia until the diagnosis of factitious hypoglycemia is firmly established (Table 3). ${ }^{6-9}$

The frequency of factitious hypoglycemia is estimated to be approximately 4 cases per $1,000,000$ in the general population, an incidence similar to that of insulinoma. ${ }^{1,8}$ It is most common among young women and typically includes patients who are knowledgeable about diabetes mellitus or who are associated with the medical profession. As stated previously, it is one aspect of the Munchausen syndrome, and afflicted patients can exert considerable and continued efforts seeking hospitalization.

The prompt and accurate differential diagnosis of factitious hypoglycemia is important for a number of reasons ${ }^{1-3,9-11}$ :

1. Factitious hypoglycemia is often difficult to diagnose with certainty.

2. Severe hypoglycemia can lead to convulsions, coma, or even death. 
3. Failure to diagnose factitious hypoglycemia can lead to extensive and costly procedures, including surgery, in the search for a nonexistent insulinoma.

4. Long-term psychiatric treatment and supportive help are necessary.

5. The diagnosis of factitious hypoglycemia carries a poor long-term prognosis with a high fatality rate.

\section{Prognosis}

Long-term follow-up is usually not available for these patients because of their tendency to physician shop. Most of these patients undergo multiple and repeated diagnostic testing and even invasive procedures and surgeries. There is a higher incidence of suicide among this population, and depression, personality disorders, and self-mutilation syndrome are often observed. The generally poor prognosis for these patients underscores the importance of early recognition of factitious disorders.

\section{References}

1. Folks DG. Munchausen's syndrome and other factitious disorders. Neurol Clin 1995;13:267-81.

2. Kaminer Y, Robbins DR. Insulin misuse: a review of an overlooked psychiatric problem. Psychosomatics 1989;30:19-24.

3. Grunberger G, Weiner JL, Silverman R, Taylor $S, \stackrel{\mathscr{N}}{\stackrel{\mathscr{N}}{\circ}}$ Gorden P. Factitious hypoglycemia due to surrepti-T tious administration of insulin. Diagnosis, treat- 3 ment, and long-term follow-up. Ann Intern Med 1988;108: 252-7.

4. Klonoff DC, Barrett BJ, Nolte MS, Cohen RM, Wyderski R. Hypoglycemia following inadvertent and factitious sulfonylurea overdosages. Diabetes Care 1995;18:563-7.

5. Cryer PE. Hypoglycemia of obscure cause. Hosp Pract Off Ed 1992;27:119-32, passim.

6. Wallach J. Laboratory diagnosis of factitious disor- $\vec{\circ}$ ders. Arch Intern Med 1994;154:1690-6.

7. Comi RJ. Approach to acute hypoglycemia. En- N docrinol Metab Clin North Am 1993;22:247-62.

8. Service FJ. Hypoglycemia. Endocrinol Metab Clin? North Am 1997;26:937-55.

9. Sener A, Gillet C, Verhelst J, DeBoeck K, Mahler C, $\frac{N}{\omega}$ Malaisse WJ. Factitious hypoglycaemia documented $\omega$ by a modified assay for the measurement of plasma $\subseteq$ sulphonylurea. Diabet Med 1995;12:433-5.

10. Braithwaite SS, Eatherton JK, Ellos WJ, Emanuele MA, Morrissey M, Sizemore GW. The ethics of sur- $\mathcal{S}$ reptitious diagnostics for factitious hypoglycemia. $\vec{\theta}$ Clin Ethics 1990;1:116-22.

11. Roy M, Roy A. Factitious hypoglycemia. An 11-year follow-up. Psychosomatics 1995;36:64-5. 